\title{
Parallel electric field generation by Alfvén wave turbulence
}

\author{
N. H. Bian, E. P. Kontar, and J. C. Brown \\ Department of Physics \& Astronomy, University of Glasgow, G12 8QQ, UK \\ e-mail: nbian@astro.gla.ac.uk \\ Received 12 January 2010 / Accepted 4 June 2010

\begin{abstract}
Aims. This work aims to investigate the spectral structure of the parallel electric field generated by strong anisotropic and balanced Methods. We consider anisotropic Alfvénic fluctuations in the presence of a strong background magnetic field. Exploiting this anisotropy, a set of reduced equations governing non-linear, two-fluid plasma dynamics is derived. The low- $\beta$ limit of this model is used to follow the turbulent cascade of the energy resulting from the non-linear interaction between kinetic Alfvén waves, from the large magnetohydrodynamics (MHD) scales with $k_{\perp} \rho_{\mathrm{s}} \ll 1$ down to the small "kinetic" scales with $k_{\perp} \rho_{\mathrm{s}} \gg 1$, $\rho_{\mathrm{s}}$ being the ion sound gyroradius.

Results. Scaling relations are obtained for the magnitude of the turbulent electromagnetic fluctuations, as a function of $k_{\perp}$ and $k_{\|}$, showing that the electric field develops a component parallel to the magnetic field at large MHD scales.

Conclusions. The spectrum we derive for the parallel electric field fluctuations can be effectively used to model stochastic resonant acceleration and heating of electrons by Alfvén waves in solar flare plasma conditions
\end{abstract} \\ Alfvénic turbulence in relation with the problem of electron acceleration from the thermal population in solar flare plasma conditions.
}

Key words. Sun: corona - Sun: flares - Sun: X-rays, gamma rays - turbulence

\section{Introduction}

Solar flares provide many challenges for crucial aspects of high energy astrophysics, including energy release, particle acceleration and transport in magnetized plasmas (e.g. Aschwanden 2002; Brown et al. 2006, as recent reviews). The impulsive phase of a flare marks the rapid release and conversion of a large amount of magnetic energy, stored in the solar corona, into the kinetic energy of particles. In the standard thick-target model, (Brown 1971; Syrovatskii \& Shmeleva 1972; Lin \& Hudson 1976), reviewed by (Brown et al. 2003; Brown \& Kontar 2005), the stream of fast electrons which emits bremsstrahlung hard $\mathrm{X}$-rays heats the dense chromospheric plasma collisionally, is produced first in the tenuous corona by electron acceleration from thermal energies $(\lesssim 1 \mathrm{keV})$ to deka-keV and MeV energies. This standard geometry of flare electron acceleration and transport is consistent with a variety of spatially resolved observations (Aschwanden et al. 2002; Emslie et al. 2003; Kontar et al. 2008; Krucker \& Lin 2008) and by electron time of flight effects in Hard X-ray light curves (Aschwanden 2002). However, an electron beam undergoing solely collisional energy loss, as in the standard thick target model, gives up around $10^{5}$ times energy to heat than to bremsstrahlung and demands (Brown 1971) a very high electron production rate to yield observed hard X-ray fluxes. Furthermore the electron beam and hard X-ray source anisotropies in the standard thick target model (Brown 1972) are much higher than inferred from the flare hard X-ray data (Kontar \& Brown 2006). Brown et al. (2009) have proposed that if fast electrons, on reaching the chromosphere, undergo re-acceleration by current sheets there, their enhanced lifetimes increase the hard X-ray yield per electron, so reducing the injection rate needed for hard X-ray production, while greatly reducing the fast electron anisotropy in the main hard X-ray source. Therefore, any mechanism that can re-accelerate electrons in the chromosphere is also of interest.

Various acceleration mechanisms have been proposed for energetic solar particles (Aschwanden 2002), including acceleration by a large scale parallel electric field (Holman 1985), electric fields inside current sheets (Litvinenko 2003; Wood \& Neukirch 2005; Bian \& Tsiklauri 2008; Siversky \& Zharkova 2009), collapsing trap acceleration (Bogachev \& Somov 2007) as well as turbulent non-resonant (Bykov \& Fleishman 2009), and resonant acceleration by waves (see the reviews by Miller et al. 1997; Petrosian 1999). Parallel acceleration between electrons and the parallel electric field produced by turbulent Alfvén waves is the subject of the present study.

The resonant coupling between a given electromagnetic mode characterized by its dispersion relation $\omega k$ and an electron gyrating at the gyrofrequency $\omega_{\mathrm{ce}}=q B_{0} / \mathrm{m}$ while streaming at the speed $v_{\|}$along the magnetic field, is given by the Doppler resonance condition, $\omega-s \omega_{\text {ce }} / \gamma=k_{\|} v_{\|}$. In this expression, $k_{\|}$ is the parallel wavenumber of the wave, $\gamma$ is the Lorentz factor and $s$ is the harmonic number of $\omega_{\text {ce }}$. Basically, the resonance condition specifies under which condition this electron experiences an electromagnetic force which is stationary. Therefore, if a broad spectrum of the electromagnetic field fluctuations associated with a particular mode is present, and moreover, if the resonance condition with this mode is satisfied for thermal electrons, then it is possible for these electrons to achieve a large energy gain, only limited by the final energy which corresponds to the last resonance with this mode. Within quasilinear theory, this resonant acceleration process is a diffusion in velocity space, from the thermal velocity $V_{\mathrm{Te}}$ up to the final velocity $V_{\mathrm{f}}$. The most straightforward way of producing a stream of fast electrons 
accelerated along the magnetic field lines is through wave resonance satisfying the condition

$\omega=k_{\|} v_{\|}$,

either by the parallel electric force $F=q E_{\|}$or the magnetic mirror force $F=\mu \nabla_{\|} B, \mu=m v_{\perp}^{2} / 2 B_{0}$ being the magnetic moment.

Many people starting from (Fermi 1949) considered stochastic acceleration of particles. Miller et al. (1996) have developed a model of thermal electron acceleration during flares based on the Landau resonance between these electrons and the fluctuating parallel mirror force produced by the compressive magnetic field component of turbulent magnetoacoustic waves. The mechanism being the magnetic analog of Landau damping is called transittime damping. Since magnetoacoustic waves have similar speeds as Alfvén waves, their frequency being given by $\omega=k V_{\mathrm{a}}$ they indeed can resonate with a population of thermal electrons, i.e. $V_{\mathrm{Te}} \sim V_{\mathrm{A}}$. Under typical plasma conditions in the solar corona (e.g. Emslie et al. 2003; Kontar et al. 2008), i.e. magnetic field $B_{0} \approx 100 \mathrm{G}$, plasma density $n \approx 5 \times 10^{9} \mathrm{~cm}^{-3}$, and electron temperature $T_{\mathrm{e}} \approx 10^{6} \mathrm{~K}$, the Alfvén velocity $\left(V_{\mathrm{A}} \sim 3 \times 10^{8} \mathrm{~cm} / \mathrm{s}\right)$ is close to the electron thermal speed $\left(V_{\mathrm{Te}} \sim 4 \times 10^{8} \mathrm{~cm} / \mathrm{s}\right)$. In the model by Miller et al. (1996), the broad spectrum of magnetic fluctuations is produced by isotropic MHD turbulence.

As stated above, the Landau resonance (1) is well satisfied between thermal electrons and shear-Alfvén waves with frequency given by $\omega=k_{\|} V_{\mathrm{A}}$. However, it is often assumed in the literature that the shear-Alfvén mode lacks the parallel electric field necessary to accelerate the particles. This is only true if non-MHD effects are ignored in the range of wavenumbers where the wave has a frequency $\omega \sim k_{\|} V_{\mathrm{A}}$. In this study, we reconsider the possibility of electron acceleration through the Landau resonance with the fluctuating parallel electric force produced by Alfvénic turbulence. This is done by investigating the spectral structure of the parallel electric field fluctuation resulting from kinetic Alfvén wave (KAW) turbulence, the KAW mode having a frequency given by $\omega=k_{\|} V_{\mathrm{A}}\left(1+\rho_{\mathrm{s}}^{2} k_{\perp}^{2}\right)^{1 / 2}$ where $\rho_{\mathrm{S}}$ is the ion-sound gyro radius. Following the same lines as the Goldreich-Sidhrar theory for Alfvénic turbulence (Goldreich \& Sridhar 1995), we derive an expression for the parallel electric field spectrum, for strong anisotropic KAW turbulence, from the large MHD scales with $k_{\perp} \rho_{\mathrm{s}} \ll 1$ down to the small "kinetic" scales with $k_{\perp} \rho_{\mathrm{s}} \gg 1$. It is shown that the magnitude of the the parallel electric field fluctuation, being an increasing function of wave number in the MHD regime but a decreasing function of the wave number in the "kinetic" regime, it reaches a maximum at the boundary, where the Alfvén wave becomes dispersive. This means that the condition $k_{\perp} \rho_{\mathrm{s}} \gg 1$ does not have to be satisfied for stochastic acceleration by Alfvén waves to be effective.

\section{Two-fluid plasma dynamics}

The starting point is a reduced set of equations, describing anisotropic two-fluid plasma dynamics in a strong magnetic field. Under the assumption of quasi-neutrality and considering that the bulk plasma electrons have negligibly small inertia, the fluid equations of motion for the ions and the electrons are

$n m_{\mathrm{i}}\left(\partial_{\mathrm{t}} \boldsymbol{V}_{\mathrm{i}}+\boldsymbol{V}_{\mathrm{i}} \cdot \nabla \boldsymbol{V}_{\mathrm{i}}\right)=-\nabla P_{\mathrm{i}}+n e\left(\boldsymbol{E}+\boldsymbol{V}_{\mathrm{i}} \times \boldsymbol{B}\right)$,

$0=-\nabla P_{\mathrm{e}}-n e\left(\boldsymbol{E}+\boldsymbol{V}_{\mathrm{e}} \times \boldsymbol{B}\right)$,

where $n$ is the plasma number density, $\boldsymbol{V}_{\mathrm{i} / \mathrm{e}}$ is the ion/electron velocity, $m_{\mathrm{i}}$ is the ion mass, $P_{\mathrm{i}, \mathrm{e}}$ the ion/electron pressure, $\boldsymbol{E}$ is the electric field and $\boldsymbol{B}$ the magnetic field. The system should be supplemented by Maxwell's equations: $\nabla \times \boldsymbol{E}=-\partial_{\mathrm{t}} \boldsymbol{B}, \nabla \times \boldsymbol{B}=$ $\mu_{0} \boldsymbol{j}$ and $\nabla . \boldsymbol{B}=0$ with $\boldsymbol{j}=n e\left(\boldsymbol{V}_{\mathrm{i}}-\boldsymbol{V}_{\mathrm{e}}\right)$.

These equations are made dimensionless by introducing a typical length scale $L_{0}$, density $n_{0}$, a typical value for the magnetic field $B_{0}$, corresponding to the Alfvén velocity $V_{\mathrm{A}}=$ $B_{0} / \sqrt{\left(\mu_{0} n m_{\mathrm{i}}\right)}$, a time scale $L_{0} / V_{\mathrm{A}}$ and the pressures are normalized to the magnetic pressure $B_{0}^{2} / \mu_{0}$. Equations (1)-(2) are then combined to give an ion equation of motion

$\partial_{\mathrm{t}} \boldsymbol{V}+\boldsymbol{V} . \nabla \boldsymbol{V}=-\nabla P+\boldsymbol{j} \times \boldsymbol{B}$,

with $P \equiv P_{\mathrm{i}}+P_{\mathrm{e}}$, while the electron equation of motion is equivalent to the generalized Ohm's law,

$\boldsymbol{E}+\boldsymbol{V}_{\mathrm{e}} \times \boldsymbol{B}=-d_{\mathrm{i}} \nabla P_{\mathrm{e}}$,

with $\boldsymbol{V}_{\mathrm{e}}=\boldsymbol{V}-d_{\mathrm{i}} \boldsymbol{j}$ and $\boldsymbol{V} \equiv \boldsymbol{V}_{\mathrm{i}}$.

Ohm's law (5) involves the non-dimensional parameter $d_{\mathrm{i}}$ which is the normalized ion skin depth $d_{\mathrm{i}} \equiv\left(c / \omega_{\mathrm{pi}}\right) / L_{0}$ with $\omega_{\mathrm{pi}}=\sqrt{\left(n \mathrm{e}^{2} / \epsilon_{0} m_{\mathrm{i}}\right)}$. The expression for its magnetic field aligned component,

$E_{\|}=-d_{\mathrm{i}} \nabla_{\|} P_{\mathrm{e}}$

shows that a parallel electric field can be produced by the electron pressure gradient along the magnetic field lines. We emphasize that this electric field is parallel to the total magnetic field, comprising the background plus its perturbation.

The existence of a strong background magnetic field $B_{0} z$ makes the plasma dynamics anisotropic with $\alpha \equiv k_{\|} / k_{\perp} \ll 1$. We can write the normalized magnetic field as $\boldsymbol{B}=\boldsymbol{z}+\delta \boldsymbol{B}$ and make the following ordering $\delta B \sim \alpha$ for its perturbation. The solenoidal condition for the magnetic field perturbation allows its perpendicular component to be written in term of a flux function: $\delta \boldsymbol{B} \simeq \nabla \psi \times z+b_{z} z$. In the same way, the perpendicular velocity is written in term of a stream function: $\boldsymbol{V} \simeq \nabla \phi \times z+v_{z} z$ with the ordering $V \sim \delta B$. Following the same standard procedure as is employed to obtain reduced magnetohydrodynamics (RMHD) from the MHD equations (Kadomtsev \& Pogutse 1974; Strauss 1976), the two-fluid Eqs. (4)-(5) yield, to order $\alpha^{2}$ in the asymptotic expansion,

$\partial_{\mathrm{t}} \psi=\partial_{z}\left(\phi-d_{\mathrm{i}} b_{z}\right)+\left[\phi-d_{\mathrm{i}} b_{z}, \psi\right]$,

$\partial_{\mathrm{t}} b_{z}=\partial_{z}\left(v_{z}-d_{\mathrm{i}} j_{z}\right)+\left[v_{z}-d_{\mathrm{i}} j_{z}, \psi\right]+\left[\phi, b_{z}\right]-\nabla . \boldsymbol{V}$,

$\partial_{\mathrm{t}} \omega_{z}=\partial_{z} j_{z}+\left[j_{z}, \psi\right]+\left[\phi, \omega_{z}\right]$

$\partial_{\mathrm{t}} v_{z}=\partial_{z} b_{z}+\left[b_{z}, \psi\right]+\left[\phi, v_{z}\right]$

where the notation $[A, B]=z \cdot(\nabla A \times \nabla B)$ is adopted and $j_{z}=$ $-\nabla^{2} \psi, \omega_{z}=-\nabla^{2} \phi$ are respectively the $z$-component of the current and the vorticity. In the reduction scheme, the fast time scale is the propagation time of the fast magnetoacoustic mode, which is therefore eliminated, while the low-frequency dynamics of the shear Alfvén and slow magnetoacoustic modes are retained. The above system can be closed by the pressure equation,

$\partial_{\mathrm{t}} p=[\phi, p]-\beta \nabla . V$,

with $p$ the normalized pressure perturbation. The plasma pressure parameter is defined as $\beta=C_{\mathrm{s}}^{2} / V_{\mathrm{A}}^{2}=\Gamma P_{0} /\left(B_{0}^{2} / \mu_{0}\right)$, with $\Gamma$ the ratio of specific heats and $P_{0}$ the background reference pressure.

In the limit $\beta \gg 1$, it can be seen from (11) that the plasma flow becomes incompressible, $\nabla \cdot V=0$, and hence, the above 
system is equivalent to the incompressible reduced Hall-MHD equations derived, for instance, by Gómez et al. (2008). For $d_{\mathrm{i}}=0$, the classical reduced-MHD equations are recovered with Eqs. (7) and (9) forming an independent system describing the non-linear dynamics of shear Alfvén waves.

Assuming $\boldsymbol{V}=0$ in the previous model leads to the reduced electron-MHD (EMHD) equations which conserve the magnetic energy $E=\int \mathrm{d}^{3} r\left[(\nabla \psi)^{2}+b_{z}^{2}\right]$. Its linear modes are the whistler waves with $\omega_{ \pm}= \pm k_{\|} d_{\mathrm{i}} k_{\perp}$. Some key properties of the EMHD turbulence have been investigated both numerically and theoretically, suggesting that the Kolmogorov type arguments work fine (Biskamp et al. 1999; Ng et al. 2003; Cho \& Lazarian 2004, 2009). A calculation along the lines of the one below for KAWs, shows that the energy spectrum for whistler wave turbulence is $E_{k_{\perp}} \propto \epsilon^{2 / 3} d_{\mathrm{i}}^{-2 / 3} k_{\perp}^{-7 / 3}$.

Relaxing the assumption of a large $\beta$, we can allow for the effect of a finite plasma compressibility. Since the perpendicular pressure balance, $\nabla_{\perp}\left(p+b_{z}\right)=0$, is satisfied to order $\alpha$ in the expansion of the ion equation of motion, the compression term $\nabla \cdot V$ can be eliminated from (11) and (8) using the fact that $p \simeq$ $b_{z}$. Therefore, defining $Z=b_{z} / c_{\beta}, c_{\beta}=\sqrt{\beta / 1+\beta}$ and $d_{\beta}=c_{\beta} d_{i}$, the following model is obtained:

$\partial_{\mathrm{t}} \psi=\partial_{z}\left(\phi-d_{\beta} Z\right)+\left[\phi-d_{\beta} Z, \psi\right]$

$\partial_{\mathrm{t}} Z=\partial_{z}\left(c_{\beta} v_{z}-d_{\beta} j_{z}\right)+\left[c_{\beta} v_{z}-d_{\beta} j_{z}, \psi\right]+[\phi, Z]$

$\partial_{\mathrm{t}} \omega_{z}=\partial_{z} j_{z}+\left[j_{z}, \psi\right]+\left[\phi, \omega_{z}\right]$

$\partial_{\mathrm{t}} v_{z}=c_{\beta} \partial_{z} Z+\left[\phi, v_{z}\right]+c_{\beta}[Z, \psi]$.

The system conserves the total energy $E=\int \mathrm{d}^{3} r\left[(\nabla \phi)^{2}+v_{z}^{2}+\right.$ $\left.(\nabla \psi)^{2}+Z^{2}\right]$. More details concerning the derivation of this reduced two-fluid MHD model can be found in (Bian \& Tsiklauri 2009).

\section{Kinetic Alfvén turbulence}

For $\beta \ll 1$, the parallel flow dynamics decouples in the above reduced two-fluid MHD model, hence $Z=-\rho_{\mathrm{s}} \omega_{z}$, and therefore it simplifies to:

$\partial_{\mathrm{t}} \psi=\partial_{z}\left(\phi+\rho_{\mathrm{s}}^{2} \omega_{z}\right)+\left[\phi+\rho_{\mathrm{s}}^{2} \omega_{z}, \psi\right]$,

$\partial_{\mathrm{t}} \omega_{z}=\partial_{z} j_{z}+\left[\phi, \omega_{z}\right]+\left[j_{z}, \psi\right]$

with $\rho_{\mathrm{s}}=C_{\mathrm{s}} / \omega_{\mathrm{ci}}$ being the ion sound gyroradius. The total energy takes the form:

$E=\int \mathrm{d}^{3} r\left[(\nabla \phi)^{2}+(\nabla \psi)^{2}+\rho_{\mathrm{s}}^{2} \omega_{z}^{2}\right]$

Notice that this model is very similar to the EMHD system when $k_{\perp} \rho_{\mathrm{S}} \gg 1$, with the compressibility effect retained, while it reduces to the standard RMHD description of shear Alfvén waves perturbations for $k_{\perp} \rho_{\mathrm{s}} \ll 1$. Linearizing this two-field model yields the frequency of the kinetic Alfvén wave:

$\omega_{ \pm}= \pm k_{\|} \sqrt{1+\rho_{\mathrm{s}}^{2} k_{\perp}^{2}}$.

This shows that the low-frequency Alfvén wave, with a frequency much smaller that the ion cyclotron frequency $\omega<\omega_{\text {ci }}$, becomes dispersive when the wavelength perpendicular to the background magnetic field is comparable or smaller than the ion sound gyroradius $\rho_{\mathrm{s}}$, i.e. $\omega_{ \pm}= \pm k_{\|} \rho_{\mathrm{s}} k_{\perp}$, this dispersion being similar to the one of the low-frequency whistler wave. We further notice that the model given by Eqs. (16)-(17) is the simplest subset of the so-called electromagnetic gyrofluid models (see e.g. Waelbroeck et al. 2009, and references therein) which are obtained as moments of the drift-kinetic equations.

From Eqs. (16)-(17), a theory for KAW turbulence is now constructed along the same lines as the Goldreich-Sidrar theory (Goldreich \& Sridhar 1995) for Alfvén wave turbulence (Kraichnan 1965). Some form of dissipation at small scales, balancing the energy input at large scales, is necessary for a steady cascade of energy to take place. It is assumed that the turbulent fluctuations are composed of KAWs, hence,

$\phi=\psi\left(\sqrt{\frac{1}{1+\rho_{\mathrm{s}}^{2} k_{\perp}^{2}}}\right)$.

Focusing first on the perpendicular cascade, we can express the energy per wave number $E_{k_{\perp}}$ from (18) and use (20) to obtain that

$E_{k_{\perp}} \propto k_{\perp} \psi_{k_{\perp}}^{2}$

Moreover, we adopt the standard assumption that the flux of turbulent energy at a given scale is determined by the turbulence at that scale and is a constant equal to the energy injection rate $\epsilon$. Therefore, the expression for the energy cascade rate is

$\epsilon \sim k_{\perp} E_{k_{\perp}} / \tau_{\mathrm{NL}}$

with the non-linear time scale being given by $\tau_{\mathrm{NL}} \sim 1 / k_{\perp}^{2} \phi_{k_{\perp}}(1+$ $\rho_{\mathrm{s}}^{2} k_{\perp}^{2}$ ), which, using (20), is equivalently expressed as

$\tau_{\mathrm{NL}} \sim \frac{1}{k_{\perp}^{2} \psi_{k_{\perp}} \sqrt{1+\rho_{\mathrm{s}}^{2} k_{\perp}^{2}}}$

Combining relations (21)-(23) yields the scaling law for the energy spectrum:

$E_{k_{\perp}}=C \epsilon^{2 / 3} k_{\perp}^{-5 / 3}\left(1+\rho_{\mathrm{s}}^{2} k_{\perp}^{2}\right)^{-1 / 3}$

where $C$ is a constant of the order of unity (Kraichnan 1965). This expression recovers the spectrum of Alfvenic turbulence, in the limit $k_{\perp} \rho_{\mathrm{s}} \ll 1$, i.e., $E_{k_{\perp}} \sim C \epsilon^{2 / 3} k_{\perp}^{-5 / 3}$, while in the dispersive range, for $k_{\perp} \rho_{\mathrm{s}} \gg 1$, then, $E_{k_{\perp}} \sim C \epsilon^{2 / 3} \rho_{\mathrm{s}}^{-2 / 3} k_{\perp}^{-7 / 3}$. Implicit in the derivation of (24) is the assumption that the fraction of the energy flux of Alfvenic turbulence which is transferred from the MHD scales onto the dispersive scales is of the order unity. Notice that since from (21), the magnetic energy spectrum is $E_{k_{\perp}}=k_{\perp}^{-1} \delta B_{\perp}^{2}$, then (24) is also equivalent to the scaling relation

$\delta B_{\perp}=C^{1 / 2} \epsilon^{1 / 3} k_{\perp}^{-1 / 3}\left(1+\rho_{\mathrm{s}}^{2} k_{\perp}^{2}\right)^{-1 / 6}$.

Now, we recall a fundamental ordering used in the derivation of the two-fluid reduced MHD system (16)-(17):

$\delta B_{\perp} \sim k_{\|} / k_{\perp}$.

This ordering is not restrictive in the sense that we are interested in the inertial range and not in the outer scale of the Alfvenic turbulence, where $\delta B_{\perp}$ can be of order unity. Using (25), (26) provides the scale dependent anisotropy of the turbulence:

$k_{\|}\left(k_{\perp}\right) \sim \epsilon^{1 / 3} k_{\perp}^{2 / 3}\left(1+\rho_{\mathrm{s}}^{2} k_{\perp}^{2}\right)^{-1 / 6}$ 
which recovers the original Goldreich-Sridhar critical balance relation $k_{\|} \propto k_{\perp}^{2 / 3}$, for Alfvén wave turbulence when $k_{\perp} \rho_{\mathrm{s}} \ll 1$, while in the dispersive range, $k_{\|} \propto k_{\perp}^{1 / 3}$.

In fact, it would have been equivalent to argue, following Goldreich \& Sridhar (1995), that the anisotropy of the turbulence is fixed by the so-called critical balance condition, i.e. to assume that the characteristic non-linear decorrelation time is of the order of the inverse KAW frequency, i.e. $\omega_{\mathrm{KAW}}^{-1} \sim \tau_{\mathrm{NL}}$, with $\omega$ given by Eq. (19). The scaling relations obtained for the energy spectrum and anisotropy in the dispersive scales of kinetic Alfvén wave turbulence (Cranmer \& van Ballegooijen 2003; Howes et al. 2008; Schekochihin et al. 2009) are similar to the ones of EMHD turbulence (Biskamp et al. 1999; $\mathrm{Ng}$ et al. 2003; Cho \& Lazarian 2004, 2009). Notice however that for an EMHD Ohm's law given by $\boldsymbol{E}=d_{\mathrm{i}} \boldsymbol{j} \times \boldsymbol{B}$, whistlers do not have a parallel electric field.

Before concluding this section, few comments are due. In deriving the energy spectrum for kinetic Alfvén wave turbulence we are relying on the existing theory developed by (Goldreich \& Sridhar 1995) for strong anisotropic and balanced Alfvén turbulence. The same approach was followed by (Schekochihin et al. 2009) based on a compressible EMHD model to describe the dispersive range of Alfvén turbulence, see also (Cranmer \& van Ballegooijen 2003). It is our framework to investigate the spectral structure of the turbulent parallel electric field. This should however not suggest that there is one universal cascade of Alfvenic fluctuations. Indeed, the previous arguments are based on the assumption that the turbulence is non cross-helical. In the MHD range, the effect of cross-helicity on the cascade of the two Elsasser energies, i.e. imbalanced turbulence, was investigated by (Lithwick et al. 2007; Beresnyak \& Lazarian 2008; Chandran 2008; Perez \& Boldyrev 2009). Imbalanced turbulence is a more general situation but it is not yet clear how the imbalance affects the dispersive range. Moreover it should be mentioned that MHD and EMHD turbulence can also be dominated by weak fluctuations, see (Galtier et al. 2002; Galtier \& Bhattacharjee 2003), weak Alfvén turbulence producing different spectra and anisotropy than the case studied here. Also, it has been argued that "dynamic alignment" of velocity and magnetic fields result in spectra that are flatter than Kolmogorov (Boldyrev 2006; Mason et al. 2006; Beresnyak \& Lazarian 2006). With these restrictions in mind we can now discuss the parallel electric field spectrum of Alfvén turbulence, a potentially important issue, which to the best of our knowledge has not been investigated so-far.

\section{Parallel electric field spectrum}

As stated above, the dispersive nature of the dynamics of KAWs involves the production of an electric field perturbation $\delta \boldsymbol{E}$ which possesses a component parallel to the magnetic field. The two components of $\delta \boldsymbol{E}$ are related to the magnitude of the perpendicular magnetic field perturbation $\delta B_{\perp}$ through the relations:

$\delta E_{\perp}=\left(1+k_{\perp}^{2} \rho_{\mathrm{s}}^{2}\right)^{-1 / 2} \delta B_{\perp}$,

for the perpendicular component and

$\delta E_{\|}=k_{\|} k_{\perp} \rho_{\mathrm{s}}^{2}\left(1+k_{\perp}^{2} \rho_{\mathrm{s}}^{2}\right)^{-1 / 2} \delta B_{\perp}$,

for the parallel component. Using the scaling (25) for $\delta B_{\perp}$, it follows that

$\delta E_{\perp}=C^{1 / 2} \epsilon^{1 / 3} k_{\perp}^{-1 / 3}\left(1+\rho_{\mathrm{s}}^{2} k_{\perp}^{2}\right)^{-2 / 3}$

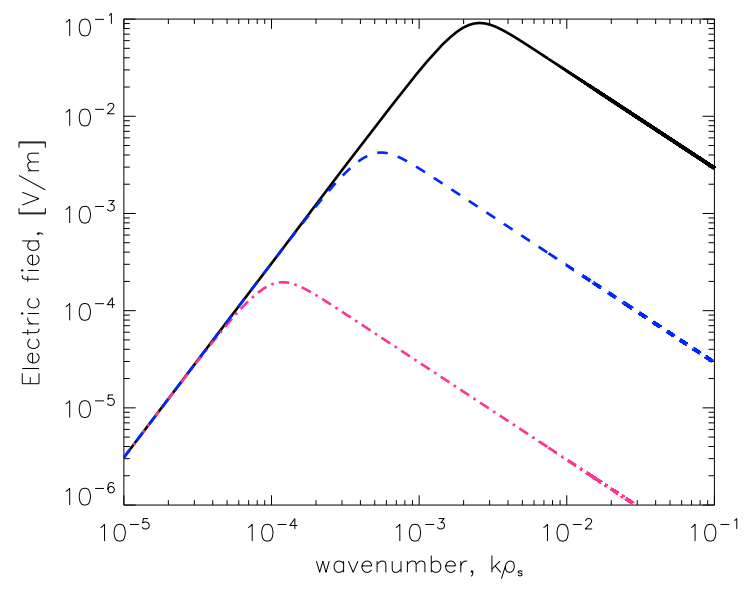

Fig. 1. Parallel electric field, $\delta E_{\|}$, as a function of $k_{\|} \rho_{\mathrm{s}}$ for different values of the large scale magnetic field perturbation $\epsilon=\left(\delta B_{\perp} / B_{0}\right)^{2}$, 1- solid black line, $10^{-2}$ - dash blue line, $10^{-4}$ - dash-dot red line.

and

$\delta E_{\|}=C^{1 / 2} \epsilon^{1 / 3} \rho_{\mathrm{s}}^{2} k_{\|} k_{\perp}^{2 / 3}\left(1+\rho_{\mathrm{s}}^{2} k_{\perp}^{2}\right)^{-2 / 3}$.

These equations, together with Eq. (27), determine the magnitude of the electric field fluctuations produced by strong anisotropic and balanced kinetic Alfvén wave turbulence as a function of wavenumber $k_{\|}$or $k_{\perp}$. Of particular interest is the scaling of the parallel electric field as a function of the parallel wave-number. It is easily shown that the parallel electric field behaves like $\delta E_{\|}\left(k_{\|}\right) \propto \rho_{\mathrm{s}}^{2} k_{\|}^{2}$ in the MHD scales when $k_{\|} \ll \epsilon^{1 / 3} \rho_{\mathrm{s}}^{-2 / 3}$ and like $\delta E_{\|}\left(k_{\|}\right) \propto \epsilon k_{\|}^{-1}$, in the dispersive scale when $k_{\|} \gg \epsilon^{1 / 3} \rho_{\mathrm{s}}^{-2 / 3}$. The magnitude of the parallel electric field, in $\mathrm{V} \mathrm{m}^{-1}$, is plotted in Fig. 1 versus $k_{\|} \rho_{\mathrm{s}}$, for different normalized energy injection rates $\epsilon=\left(\delta B_{\perp} / B_{0}\right)^{2}, \delta B_{\perp}$ being here the dimensional value of the magnetic perturbation at the injection scale (see below).

\section{Discussion and conclusions}

There exists an extensive literature on stochastic acceleration by resonant interaction between waves and particles. However, to our knowledge, a scenario for field-aligned acceleration of electrons by the parallel electric field produced by Alfvenic turbulence has not been considered so-far. The reason is that it is generally assumed that the MHD Alfvén mode, whose frequency is given by $\omega \sim k_{\|} V_{\mathrm{A}}$ when $k_{\perp} \rho_{\mathrm{s}} \ll 1$, lacks the parallel electric field to accelerate the particles. A main objective of the present work is to emphasize that, on the contrary, the role of the parallel electric field intrinsic to the Alfvén wave dynamics in a warm plasma should not be ignored, even at the scales of standard MHD. We believe that while a scenario based on Alfvén waves for electron acceleration during solar flares remains a conjecture, as is any other acceleration mechanism proposed so far, it is particulary attractive. The reason is due to the body of observational evidences on the role played by these waves in controlling many aspects of the dynamics of astrophysical plasmas.

In plasma conditions typical of solar flares, values of the fluctuating parallel electric field can be significant. We consider a range of solar plasma parameters: guiding magnetic field $B_{0}=100$ Gauss, plasma density $n_{\mathrm{e}}=5 \times 10^{9} \mathrm{~cm}^{-3}$, plasma temperature $1 \mathrm{MK}$, and the loop length scale $L_{0}$. Normalizing 
the wavenumber by $\rho_{\mathrm{s}}=C_{\mathrm{s}} / \omega_{\mathrm{ci}}$, the amplitude of the parallel electric field is presented in Fig. (1). For solar flare parameters $\rho_{\mathrm{s}} \simeq 10 \mathrm{~cm}$, the maximum electric fields $2 \times 10^{-4}-10^{-1} \mathrm{~V} \mathrm{~m}^{-1}$ appears at the scales $\lambda=\rho_{\mathrm{s}} / k_{\|} \sim 10^{5}-5 \times 10^{3} \mathrm{~cm}$. The maximum values of the electric field and the characteristic scales can be derived explicitly from the results of the previous section. The maximum of the electric field amplitude

$\delta E_{\|}^{\max } \sim \epsilon^{2 / 3} \rho^{2 / 3}$

is reached at

$k_{\|}^{\max } \sim \epsilon^{1 / 3} \rho_{\mathrm{s}}^{-2 / 3}$.

These are dependent on the magnitude of the magnetic perturbation $\epsilon=\left(\delta B_{\perp} / B_{0}\right)^{2}$ at the energy injection scale $L_{0}$.

Our estimate for the maximum electric electric field fluctuation produced by Alfvén wave turbulence show that the latter can be rather strong. Indeed, for the adopted solar flares plasma parameters, the Dreicer electric field is $\sim 0.02 \mathrm{~V} \mathrm{~m}^{-1}$, and the maximum amplitude of the fluctuating field can exceed this values.

The parallel electric force associated with the Alfvén wave dynamics could play an important role in a number of cases. First, as the primary source of thermal electron acceleration, where waves and turbulence are triggered by the reconnection process (Bellan 1998; Longcope \& Priest 2007) or as a result of the twisting of the field lines anchored in the photosphere. It has also been proposed that part of the energy released during magnetic reconnection is transported by Alfvén waves to the chromosphere (Emslie \& Sturrock 1982; Fletcher \& Hudson 2008). Therefore, in a situation where Alfvenic turbulence fills the loop, the waves will affect the transport of the energetic electrons to the chromosphere. Acceleration occurs along the field lines which are perturbed by the Alfvén dynamics, hence the pace of the acceleration along $\boldsymbol{B}=\boldsymbol{B}_{0}+\boldsymbol{\delta} \boldsymbol{B}_{\perp}$ also controls the cross$\boldsymbol{B}_{0}$ transport. Finally, as already mentioned in the Introduction, we note that if the electric field produced by Alfvén turbulence can re-accelerate non-thermal electrons injected into the chromosphere this revision of the standard Thick Target Model may resolve existing problems with it. Whether this is really the case depends on the detailed nature of the interaction between the parallel electric field and the electrons which requires a kinetic description, the subject of a future publication.

Acknowledgements. This work is supported by a STFC rolling grant (N.H.B., E.P.K., J.C.B.) and an STFC Advanced Fellowship (EPK). Financial support by the Leverhulme Trust grant (F/00179/AY) and by the European Commission through the SOLAIRE Network (MTRN-CT-2006-035484) is gratefully acknowledged.

\section{References}

Aschwanden, M. J. 2002, Space Sci. Rev., 101, 1

Aschwanden, M. J., Brown, J. C., \& Kontar, E. P. 2002, Sol. Phys., 210, 383
Bellan, P. M. 1998, Phys. Plasmas, 5, 3081

Beresnyak, A., \& Lazarian, A. 2006, ApJ, 640, L175

Beresnyak, A., \& Lazarian, A. 2008, ApJ, 682, 1070

Bian, N., \& Tsiklauri, D. 2008, A\&A, 489, 1291

Bian, N. H., \& Tsiklauri, D. 2009, Phys. Plasmas, 16, 064503

Biskamp, D., Schwarz, E., Zeiler, A., Celani, A., \& Drake, J. F. 1999, Phys. Plasmas, 6, 751

Bogachev, S. A., \& Somov, B. V. 2007, Astron. Lett., 33, 54

Boldyrev, S. 2006, Phys. Rev. Lett., 96, 115002

Brown, J. C. 1971, Sol. Phys., 18, 489

Brown, J. C. 1972, Sol. Phys., 26, 441

Brown, J. C., \& Kontar, E. P. 2005, Adv. Space Res., 35, 1675

Brown, J. C., Emslie, A. G., \& Kontar, E. P. 2003, ApJ, 595, L115

Brown, J. C., Emslie, A. G., Holman, G. D., et al. 2006, ApJ, 643, 523

Brown, J. C., Turkmani, R., Kontar, E. P., MacKinnon, A. L., \& Vlahos, L. 2009, A\&A, 508, 993

Bykov, A. M., \& Fleishman, G. D. 2009, ApJ, 692, L45

Chandran, B. D. G. 2008, ApJ, 685, 646

Cho, J., \& Lazarian, A. 2004, ApJ, 615, L41

Cho, J., \& Lazarian, A. 2009, ApJ, 701, 236

Cranmer, S. R., \& van Ballegooijen, A. A. 2003, ApJ, 594, 573

Emslie, A. G., \& Sturrock, P. A. 1982, Sol. Phys., 80, 99

Emslie, A. G., Kontar, E. P., Krucker, S., \& Lin, R. P. 2003, ApJ, 595, L107

Fermi, E. 1949, Phys. Rev., 75, 1169

Fletcher, L., \& Hudson, H. S. 2008, ApJ, 675, 1645

Galtier, S., \& Bhattacharjee, A. 2003, Phys. Plasmas, 10, 3065

Galtier, S., Nazarenko, S. V., Newell, A. C., \& Pouquet, A. 2002, ApJ, 564, L49

Goldreich, P., \& Sridhar, S. 1995, ApJ, 438, 763

Gómez, D. O., Mahajan, S. M., \& Dmitruk, P. 2008, Phys. Plasmas, 15, 102303

Holman, G. D. 1985, ApJ, 293, 584

Howes, G. G., Cowley, S. C., Dorland, W., et al. 2008, J. Geophys. Res. (Space Physics), 113, 5103

Kadomtsev, B. B., \& Pogutse, O. P. 1974, Soviet Journal of Experimental and Theoretical Physics, 38, 283

Kontar, E. P., \& Brown, J. C. 2006, ApJ, 653, L149

Kontar, E. P., Hannah, I. G., \& MacKinnon, A. L. 2008, A\&A, 489, L57

Kraichnan, R. H. 1965, Phys. Fluids, 8, 1385

Krucker, S., \& Lin, R. P. 2008, ApJ, 673, 118

Lin, R. P., \& Hudson, H. S. 1976, Sol. Phys., 50, 153

Lithwick, Y., Goldreich, P., \& Sridhar, S. 2007, ApJ, 655, 269

Litvinenko, Y. E. 2003, Adv. Space Res., 32, 2385

Longcope, D. W., \& Priest, E. R. 2007, Phys. Plasmas, 14, 122905

Mason, J., Cattaneo, F., \& Boldyrev, S. 2006, Phys. Rev. Lett., 97, 255002

Miller, J. A., Larosa, T. N., \& Moore, R. L. 1996, ApJ, 461, 445

Miller, J. A., Cargill, P. J., Emslie, A. G., et al. 1997, J. Geophys. Res., 102, 14631

Ng, C. S., Bhattacharjee, A., Germaschewski, K., \& Galtier, S. 2003, Phys. Plasmas, 10, 1954

Perez, J. C., \& Boldyrev, S. 2009, Phys. Rev. Lett., 102, 025003

Petrosian, V. 1999, in Plasma Turbulence and Energetic Particles in Astrophysics, Proceedings of the International Conference, Cracow (Poland), 5-10 September, Obserwatorium Astronomiczne, Uniwersytet Jagielloński, Kraków 1999, ed. M. Ostrowski \& R. Schlickeiser, 135

Schekochihin, A. A., Cowley, S. C., Dorland, W., et al. 2009, ApJS, 182, 310

Siversky, T. V., \& Zharkova, V. V. 2009, J. Plasma Phys., 75, 619

Strauss, H. R. 1976, Phys. Fluids, 19, 134

Syrovatskii, S. I., \& Shmeleva, O. P. 1972, AZh, 49, 334

Waelbroeck, F. L., Hazeltine, R. D., \& Morrison, P. J. 2009, Phys. Plasmas, 16, 032109

Wood, P., \& Neukirch, T. 2005, Sol. Phys., 226, 73 